Die mitgetheilten Versuche wurden im Laboratorium des Herrn Professor H. Munk ausgeführt. Ich sage Herrn Professor Munk für Ueberlassung des Materials und für das Interesse, mit welchem er der Untersuchung folgte, meinen aufrichtigen Dank.

\title{
XVI. \\ Ueber einen Fall von Myosarcoma striocellulare der Niere ${ }^{1}$ ).
}

Aus dem pathologischen Institute in. Halle a. S.

Von Dr. F. Marcband, Assistenten am pathologischen Institut.

Die nachfolgenden klinischen Notizen über den seltenen Geschwulstfall, welcher den Gegenstand dieser Mittheilung bildet, verdanke ich der Freundlichkeit des behandelnden Practikanten, Herrn Stud. med. Gaudin, sowie dem Assistenten an der hiesigen chirurgischen Klinik, Herrn Dr. 0 berst.

Der Patient, Arthur Hoerner, kam am 3. Juni 1877 - damals 16 Monate alt - in Behandlung der hiesigen medicinischen Poliklinik. Die Mutter ist seit längerer Zeit kränklich, es besteht sonst jedoch keine hereditäre Krankheitsanlage. Seit etwa $\frac{3}{4}$ Jahren wollen die EItern des Kindes ein allmäbliches Dickerwerden des Unterleihes bemerkt haben, während das Kind im Uebrigen abnahm. Doch erst 6 Wochen vor dem oben angegebenen Termin war eine Geschwulst am Unterleibe des Kindes deutlicher wahroehmbar, welche seitdem, von oben nach unten wachsend, stetig zupahm, obne erbebliche Krankheitssymptome zu machen. Der Stuhlgang soll ab und zo nicht in Ordnung gewesen sein, und das Kind zeitweise Verstopfung, ein andermal Durchfall gebabt haben. Am genannten Tage zeigte sich der Leib des Kindes ziemlich stark aufgetrieben; die ganze rechte Hälfte desselben war von einer Geschwulst eingenommen, die nach Percussion und Palpation mit der Leber zusammenzubängen schien, sich fest und hart, nicht fluctuirend anfühlte. Die Dämpfung ging nach links wenig über die Mittellinie binaus, und reichte nach unten bis einige Centimeter weit unter Nabelböhle. Die Begrenzung war im Ganzea rundlich, ohne Erbabenheiten. Die Wahrscheinlichkeitsdiagnose wurde auf Echinococcus der Leber gestellt, jedoch ergaben zwei Punctionen mit der Pravaz'schen Spritze keine Flüssigkeit.

1) Das betreffende Präparat wurde in der Section für pathologische Anatomie der Gesellschaft deutscher Naturforscher und Aerzte in München demonstrirt. 
Am 14. August wurde das Find in die chirurgische Klinil anfgenommen. Es war stark apgemagert und höchst anämisch. Der Umfang des Leibes betrug dicht unter den falschen Rippen 54 , in der Mitte des Unterleibes 61, um den Nabel, efwa eine Hand breit über der Symphyse $51 \mathrm{Cm}$. Diese Ausdebnnog des Leibes war bedingt durch einen glattwandigen, unten seitlich mit flach kugiigen Prominenzen versehenen, undeutlich fluctuirenden Tumor. Derselbe war nach unten, drei Finger breit über der Symphyse, wohl abzugrenzen, und in toto etwas beweglich; die Palpation nicht schmerzhaft. Unter dem Rippenhogen war die Percussion (links?) schwach tympanitisch, zu beiden Seiten und im Mesogastrium leer; die pralle Spannung der Geschwulst besonders in der seitlichen Bauchgegend ausgeprägt.

Die Diagnose des Herrn Geheimrath Volkmann lautete auf Sarcom der Lymphdrüsen oder der Niere.

Am 16. August wurde das Kind als unheilbar entlassen.

Seitdem veränderte sich sein Zustand wenig, doch nahm der Umfang des Leibes noch etwas zu, währeod die Kräfte mehr und mebr schwanden, his am 13. September Morgens 7 Uhr der letale Ausgang erfolgte.

Section an demselben Tage, 3 Uhr Nachmittags.

Sehr abgemagerte Leiche von $75 \mathrm{~cm}$. Länge, mit sehr blasser Haut. Abdomen und unterer Theil des Thorax sebr stark aufgetrieben, von praller, stellenweise undeutlich fluctuirender Consistenz. Grösster Unfang $62,5 \mathrm{Cm}$.

Bei Eröffnung des Abdomen liegt zanächst ein glattwandiger Tumor vor, welcher beinahe die ganze sehr erweiterte Bauchböhle ausfüllt. Am unteren Rande desselben liegt das Colon, darunter einige Dünndarmschlingen, am oberen Umfange die Leber, welche in der Mittellinie das Sternaw um $5 \mathrm{Cm}$. ïberragt. Der vorliegende Theil derselben ist der rechte Lappen, während der linke vollständig im linken Hypochondrium gelagert ist; die Insertion des Lig. teres findet sich etwa in der linken Mammillarlinie.

Stand des Zwerchfells rechts an der 3., links an der 4. Rippe.

Das Herz ist etwas quergelagert, enthält eine geringe Menge sehr dünnflïssigen Blutes, in der Pulmonalis und Aorta sehr geringe Spechhautgerinnsel; Musculatur sehr blass, sonst keine Veränderung.

Beide Lungen ausgedehnt, durchweg lufthaltig und sehr blass.

Es zeigt sich, dass der erwähnte Tumor von der rechten Nierengegend ausgeht; vom rechten Umfange desselben erstreckt sich eine bandförmige Verwachsung zum rechten Leberrande (Lig. hepatico-renale). Letztere ist anch mit dem hinteren Theile ihres rechten Lappens fest mit dem Tumor verbunden; der Lob. Spigelii dadurch abgeflacht und unkenntlich. Ueber die linke Hălfte der Geschwalst spannt sich ein Theil des Omentum (Lig. gastro-colicum), derselben fest anhaftend. Das Coecum und Colon ascendens umgiebt, von der Regio iliaca dext. an, den Rand des Tumor, und steigt in etwas lockerer Verbindung an der linken Seite desselben in die Höhe, während am linken oberen Umfange der Geschwulst das Duodenum bis zum Pylorus angebeftet ist. Der zusammengezogene Magen liegt in fast sentrechter Richtung im linken Hypochondrium, von dem linken Leberlappen ganz verdeckt. Seine Schleimbat ist stark längsfaltig, röthlichweiss, mit etwas Schleim bedeckt. 
Im Dünndarm, welcher ganz nach links und unten verdrängt ist, wenig schleimiger Inhalt; seine Wandung ohne hesondere Veränderung; im Dickdarm gallig gefärbte dünne Massen.

Die Milz, an der normalen Stelle gelegen, $10 \mathrm{Cm}$. lang, weich, blassroth; ihre Follikel gross, mit verwaschenen Grenzen.

Die Leber, $20 \mathrm{Gm}$. breit, abgeflacht, recbter Lappen im Sagitaldurchmesser 13, linker $12 \mathrm{Cm}$. Am vorderen Rande des recbten Lappens findet sich, einige Centimeter nach rechts von der Gallenblase, ziemlich in der Mitte des Leibes, ein durch die ganze Dicke der Substanz reichender, äber haselnussgrosser Geschwulstknoten von graurötllicher Farbe, we]cher sowobl anf der oheren als auf der unteren Fläche des Organs eine flache Hervorragung bildet: Auf dem Durchschnitt ist dieser Knoten ebenfalls grauroth, von markig-weicher Beschaffenheit; von der Schnittfäche lässt sich trüber Saft abstreichen. Ein ganz ähnlicher Knoten mit flacher centraler Vertiefuog, etwa groschengross, fiudet sich an der unteren Fläche des linken Lappens.

Das Parenchym der Leber ist feucht, blass gelbbrann, die Läppchen etwas undeutlich. In der Gallenblase blassgelbe Galle.

Das Pancreas ist aufiallend gross $(13 \mathrm{Cm}$.), aber sonst obne Veränderung. Die Mesenterialdrüsen leicht geschwollen, weich.

Die linke Niere $8 \mathrm{Cm}$. lang, entsprechend breit und dick, sehr blass, von durchscheinender graugelblicher Forbe, ziemlich fester Consistenz; sowohl Mark als Rinde von derselben Färbung. L. Nebenniere ohne Veränderung.

An Stelle der rechten Niere findet sich der Tumor, welcher an der rechten Seite der Lendenwirbelsäule und in der rechten Weiche, nach oben bis zum Zwerchfell, nach unten auf der Beckenfascie angewachsen, aber überall leicht abzulösen ist. Er besitzt eine Jänglichrunde Gestalt, und ist mit seinem grössten Durchmesser quergelagert, so dass das linke Ende unter dem linken Rippenbogen liegt.

Der Tumor misst in der grössten Ausdebnung $22 \mathrm{~cm}, 14 \mathrm{Cm}$. in der Dicke nnd $13-14$ in der Höhe. Das Gewicht beträgt $2770 \mathrm{Grm}$.

Er zerfällt in mehrere rundliche, durch flache Einschnitte getrennte apfel- bis faustgrosse Knollen, weiche namentlich an seiner hinteren Fläche stark ausgebildet sind, während die nach vora gekehrte, vom Peritoneum überzogene Fläche glatt und ebener ist. Die larbe ist gelblich- bis röthlichweiss, an mehreren steilen verlaufen ziemlieb weite Gefässe. An dem hinteren Unfange, welcher eine Art Hilus zeigt, findet sich, ziemlich in der. Mitte des unteren Randes, ein wohl erhaltener Theil der Niere, welche frei herrorragt, aber mit der Geschwulst fest verbunden ist. Die Substanz dieses nngefähr die Hälfte der normalen betragenden Nierenabschnittes ist blass-gelbliclugrau, derb. Der übrige Theil der Niere ist flach ausgebreitet und stellt eine allmählich verdünnte Schicht dar, welche sich zwar als Nierensubstanz deutlich erkennen lässt, aber doch ohne scharfe Grenze allmählich aufhört. Weiter nach ohen ragt noch ein kleiner Abscbnitt des oberen Endes der Niere hervor. Neben demselben liegt die verhältnissmässig grosse Nebenviere flach ausgebreitet auf dem oberen Umfange der Geschwulst, mit dieser durch Bindegewebe fest verlöthet. Art. nnd V. renalis sind erhalten, aber beträclutich verlän- 
gert; auch der Ureter ist bis zum Niereubecken durchgängig. Das letztere ist entsprechend der veränderten Gestalt der Niere auseinandergezogen, erweitert; es entbält einen umfangreichen, aus der Tiefe des Hilus hervorragenden polypösen Körper von etwa Fingerdicke und $5 \mathrm{Cm}$. Länge, welcher vielfacb, namentlich an seinem freien Ende, mit röthlichen, weichen, papillären Wucherungen besetzt ist. Auch von der benachbarten Wand des Beckens gehen ähnliche Wucherungen aus, während der dem freien Nierenabschnitt angehörende Theil die gewöhnliche Beschaffenheit besitzt.

Die Geschwolst besitzt eine fleischige, stellenweise täuschend Fluctuation darbietende Consistenz. Auf dem Durchschnitt besteht dieselbe aus mebreren rundlich keilförmigen Abtheilungen, den grossen Knollen der Oberfläche entsprechend. Die einzelnen Lappen oder Abtheilungen sind durch Bindegewebssepta getrennt. Die Farbe der Schnittfäche der Hauptmasse ist im Ganzen gelblichweiss. Eine der Hauptabthellungen des Tumor, von Faustgrösse, welche das linke Ende desselben einnimmt, ist auf dem Durchschnitt grösstentheils erweicht, trübe grauroth, in der Peripherie jedoch derber und faserig. In der nächsten Nähe finden sich in der Tiefe der Geschwulst einige mehr gelbgraue, ebenfalls weiche, doch mehr homogene Abtheilungen; die Hauptmasse der Geschwulst aber besteht aus vielfach unter einander verflochtenen Fasermassen, welche hier und da rundliche Abtheilungen von mehr gelber oder gerötheter Farbe einschliessen. Die in der Längsrichtung durchschnittenen Faserbündel besitzen ein glänzend weisses fast sehniges Aussehen; die Consistenz des. Gewebes ist elastisch und zähe. Beim Durchschneiden fliesst etwas schleimige Flüssigkeit ab. An einigen der rundlichen Knollen kommen unter einer äusseren Schale spaltförmige durch weisse gelappte Massen begrenzte Lücken zum Vorschein, welche schleimige Flüssigkeit enthalten. Die verdickte Nierenkapsel überziebt continuirlich die ganze Geschwulst, deren Oberfläche nach Abzug der Kapsel glatt zurückbleibt. Auf dem Durchschnitt grenzt sich die Geschwulst gegen die Nierensubstanz durch eine dicke fibröse Schicht vollkommen scharf ab.

Die mikroskopische Untersuchning ergiebt, dass die Hauptmasse der Geschwulst aus quergestreiften Muskelfasern besteht, indess entspricht der angedeuteten makroskopischen Verschiedenheit der einzelnen Theile auch ein verschiedenes bistologisches Verhalten; der weiche bräunliche oder graurothe Theil der Geschwulst, welcher in seiner Mitte in Zerfall begriffen ist, ähnelt am meisten den kleinzelligen Sarcomen, und Beimischungen der letzteren Art finden sich auch an einzelnen anderen Stellen der Geschwulst. Es bandelt sich also um ein Myoma striocellulare sarcomatosum (Myosarcoma striocellulare).

Die myomatösen Theile der Geschwulst zeichnen sich durch ihre deutliche faserige, fleiscbige Beschaffenheit aus, welche ganz an die der grossen Dterusmyome erinnert, doch ist der stellenweise fast seidenartige Glanz auffallend. Diese ganze Fasermasse ist ansammengesetzt aus langen Bündeln quergestreifter Muskelfasern, welche in ein zähes feinfibrillăres Bindegewebe eingelagert sind. Die Muskejfasern sind im Vergleich zu denen der Körpermusculatur sebr schmal, variiren aber noch beträchtlich in der Dicke $(0,003-0,007 \mathrm{Mm}$.) and sind nicht pigmentirt. Die Bündel bestehen meist aus sebr zahlreichen Fasern, welche fest zusammenhalten, jedoch nach Behandlung mit Kalilauge oder längerer Maceration in Müller'scher 
Flüssigkeit sich leicht isoliren lassen. Die Bündel durchkreazen sich in verschiedener Richtung, so dass man auf Schnitten längs-, quer- und schräg durchschnittene antrifft. Dabei variirt die bindegewebige Zwischensubstanz sehr beträchtlich, denn während sie stellenweise nur sebr geringe Zwischenräume ausfüllt, bildet sie as anderen Stellen breite Lagen, gegen welche, namentlich an der Peripherie der Geschwulst, die Muskelzüge an Zahl und Stärke allmählich zurïcktreten. In dem feinfibrillären Bindegewebe liegen zahlreiche spindelförmige Zellen, den Fasern parallel geordnet; dieselben lassen sich durch Zerzupfen isoliren, besitzen einen länglichrunden etwas platten Kern, an dessen beiden Enden die Zelle in einen mehr oder weniger langen schmalen Fortsatz ausläuft.

Zum Studium der Muskelfasern eignen sich am meisten die weicheren zellenreichen Theile der Geschwulst, in welcher man durch Zerzupfen Muskelfasern in den verschiedensten Stadien der Ausbildung erhält, indess fehlen dieselben auch in den derbfaserigen Theilen durchaus nicht. Und zwar sind erstens lange und schmale, an den Enden spitz zulaufende ziemlich glänzende Fasern mit einem länglichen Kern vorhanden. Derartige Fasern hängen zuweilen an den Enden zusammen, so dass sie also schmale Bänder darstellen, welche in gewissen Abständen Kerne tragen; zuweilen liegen zwei bis drei Kerne dicht hinter einander in einer Faser. Diese Gebilde sind entwederganz glatt, oder siezeigen, meist in der Nähedes Kernes eine beginnende $\mathbf{Q}$ uerstreifung. Daneben kommen kürzere und zugleich breitere spindelförmige Elemente mit länglichrundem Kern und 1-2 deutlichen Kernkörperchen vor, welche eine deutliche Querstreifung zeigen. Endlich finden sich etwas breitere bandförmige Fasern, welche in gewissen Abständen Kerne von der Breite der Fasern selbst tragen. Diese letzteren besitzen sämmtlich eine deutliche Streifung, indess sind auch hjer Verschiedenheiten vorhanden, indem nehmlich bei den einen die Streifung sich nur auf einen Saum zu beiden Seiten der Faser beschränkt, während der mittlere Theil frei bleibt, oder die Querstreifung erstreckt sich auf die ganze Breite der Faser. Es gelingt nicht selten, derartige Gebilde von bedeutender Länge zu isoliren, welche an einem Ende abgerissen sind, an dem anderen spitz zulaufen, andere sind auch an beiden Enden zugespitzt, stellen also lange Spindeln mit 3-4 oder mehr hinter einander liegenden Kernen vor. Schliesslich finden sich Fasern, welche die $2-3$ fache Breite der letzterwähnten besitzen und die Hauptmasse der Muskelbündel bilden. Diese zeigen sämmtlich eine deutliche Querstreifung, häufig auch eine sehr ausgesprochene Längsstreifung, welche nicht selten die erstere überwiegt, endlich eine Anzahl Kerne, deren Breite etwa die Hälfte der Faser beträgt, und welche in gewissen Abständen von einander liegen, und zwar stets an der Oberfläche. Niemals fand sich jedoch ein deutliches Sarcolemm vor.

Die Breite der Querstreifen ist etwas verschieden, in der Regel übertreffen die dunklen Streifen die hellen, zuweilen ist das Umgekehrte der Fall; eine Spaltung in Fibrillen ist häufig zu beobachten. trachten.

Das Verhalten der Kerne der jüngeren Fasern ist noch etwas genauer zu be-

Nicht selten sieht man an spindelförmigen Fasern mit quergestreifter Substanz, dass diese letztere sich in der den Kern tragenden Anschwellung gewissermaassen in zwei Fibrillen spaltet, welche etwas auseinanderweichen und den länglichrunden 
Kern 2 wischen sich lassen. An beiden Enden des letzteren bleibl ein schmaler dreieckiger Raum übrig. An anderen Fasern mit mehreren Kernen wiederholt sich dieselbe Erscheinung an jedem einzelnen; es ist dann der zwischen je zwei Kernen liegende Theil der Faser entweder ganz quergestreift, oder es erstreckt sich die Spaltung der quergestreiften Substanz auch auf diesen Theil der Faser, so dass dieser ein Band mit quergestreiftem Saume darstellt. Bei anderer Einstellung des Mikroskops kann man sich jedoch an manchen dieser Fasern überzeagen, dass die Streifung sich schwächer über die ganze Breite der Faser fortsetzt, oder es verläuft in dem mittleren Theil eine gesonderte quergestrelfte Fibrille in einer etwas anderen Ebene.

Bei Betrachtung dieser Bilder kann man leicht zu der Ansicht kommen, dass die Kerne im Innern der Faser liegen, in der That liegen sie aber stets, wie man sich überzeugt, wenn man die Faser in verschiedener Lage betrachtet, a uf derselben, so dass bei seitlicher Drebung der Faser die Kerne an der Seite frei hervorspríngen. Sie scheinen dabei durch ein zartes Häutchen, welches sich über sie binwegspannt, angeheftet. Dieses Verhältniss bleibt dasselbe während der ganzen Entwickelung der Faser. Die jüngsten spindelförmigen Elemente erscheinen von der Seite gesehen als schmale lineare glänzende Strejfen oder Fasern, an deren einer Seite der Kern aufsitzt. An den melbrkernigen längeren Fasern finden sich mehrere dieser hervorragenden Kerne hinter einander, an anderen sieht man nicht selten den einen Kern von der Seite, den anderen von der Fläche; dies Verhältniss findet sich dann bel den ausgebildeten Muskelfasern, welche an der Oberfläche verstreute platte Kerne tragen.

Die Fasern mit beiderseitigem quergestreiften Saume erscheinen von der Fläche gesehen als Halbrinnen; betrachtet man jedoch feine Querschnitte der Muskelfasern, so findet man, dass diese fast durchgängig eine rundlich polygonale Form haben; der Kern liegt stets an der einen Seite, welche zuweilen leicht eingebogen ist. Die wabre Gestalt der jungen Muskelfasern ist also rundlich prismatisch. (Zur Sicbtbarmachung der Kerne bediente ich mich mit Vortheil der Fuchsinfärbung mit nachfolgendem Auswaschen.)

Was den weichen sarcomähnlichen Theil der Geschwulst betrifft, so besteht derselbe aus dicht gedrängten kleinen Zeflen mit ruadlichen Kernen und sehr zartem feinkörnigem Protoplasma. Eine Zwischensubstanz ist vielfach nicht wahrnehmbar; zuweilen sind die Zellen verfettet. In dem Gewebe finden sich zablreiche weite, sehr dünnwandige Gefässe, obne besondere bindegewebige Scheide; häufig ist anch die zellige Masse von Blutkörperchen durchsetzt und in körnigem Zerfall begriffen, bauptsächlich in der Mitte des grösseren graurothen Knotens. An der Grenze desselben wird das Gewebe allmählich fester, die Zellen sind meist spindelförmig, die Kerne, welche sich zugleich verlängern, ordnen sich parallel zu einander und es tritt eine undeutlich fibrilläre Zwischensubstanz auf. In dem kleinzelligen Gewebe finden sich verstreut die oben bescbriebenen Anfangsstadien der Muskelfasern, anscheinend regellos durch einander; in den festeren peripherischen Thellen dagegen finden sich Bündel ausgebildeter Muskelfasern, welche allmäblich an Masse zunehmen and die weiche Geschwulst concentrisch umgeben.

In ähnlicher Weise sind auch die kleineren zellenreichen Herde beschaffen, 
welche sich verstreut in den festen Theilen der Geschwulst finden; auch diese enthalten zahlreiche junge Entwickelungsstufen von Muskelfasern.

In einigen derben Geschwulstknoten sind zwischen den Muskelbïndeln Gruppen von grossen Fettzellen vorhanden, welche sich auch makroskopisch durch die gelbliche Färbung documentiren.

In der Nähe einer solchen Stelle in der Tiefe der Geschwulst kamen auch unzweifelhafte epitheliale Bildungen vor, und zwar kleine verzweigte Schläuche, welche mit Cylinderepithel ausgekleidet waren. Stellenweise gingen diese in kleine cystische Hohlräıme über, deren Wände ebenfalls ein niedriges Cylinderepithel trugen; in einigen dieser kleinen Räume hatte auch eine Anbäufung unregelmässig polyedrischer Epithelzellen stattgefunden. Eine Tunica propria war nicht deutlich; zuweilen waren die epithelialen Bildungen von dicht gedrängten Rundzellen umgeben, so dass die Grenzen nicht deutlich festzustellen waren.

Die blumenkohlähnlichen Wucherungen im Niereabecken bestanden ebenfalls aus dicht gedrängten Rundzellen, doch fanden sich auch hier drüsenäbnliche Gebilde vor. Die Oberfläche der Wucherungen ist überzogen von einem einfachen kurz cylindrischen Epithel, mit welchem jene Gebilde zusammenhingen.

Was endlich die Knoten in der Leber betrifft, so habe ich musculöse Elemente bisher vergeblich in denselben gesucht; sie bestanden fast gänzlich aus dicht gedrängten Rundzellen mit sehr zartem Protoplasma. Diese sind eingelagert in eine fein granulirte Zwischensubstanz, welche an vielen Stellen ganz zu fehlen scheint; erst nach dem Erbärten bildete sich ein feinfädiges Netz, an anderen Stellen eine mehr homogene durchsichtige Zwischensubstanz. . Die Zellenmassen werden durchzogen von einigen schmäleren oder breiteren längsfaserigen Streifen, welche ebenfalls sebr weich, undeutlich fibrillär sind und spindelförmige Elemente einschliessen; letztere gehen an den Rändern in die dicht gedrängten Rundzellen über. An der Grenze des Knotens finden sich in demselben Reste des Lebergewebes.

Die eigenthümliche Geschwulstform, welche hier vorliegt, ist in der Niere zuerst von Eberth ${ }^{1}$ ) beobachtet worden; ein zweiter Fall wurde von Cohnheim ${ }^{2}$ ) beschrieben, ein dritter ist vor Kurzem von Landsberger ${ }^{3}$ ) bekannt gemacht und ebenfalls durch Gohnheim untersucht worden. Alle diese Fälle boten untereinander eine grosse Uebereinstimmung dar. Sämmtliche kamen bei Kindern unter anderthalb Jahren vor, besassen eine sehr bedeutende Grösse und waren rapide gewachsen. In drei Fällen war die Geschwulst doppelseitig, in dem Falle von Landsberger fand sich ausser den beiden Nierengeschwulsten noch eine dritte, als ein Adnex der rechtsseitigen Geschwulst, vor der Wirbelsäule; die Entstehung derselben beruhte wahrscheinlich auf demselben Vorgange

1) Dieses Archiv 1872. Bd. 55. S. 518.

2) Dieses Archiv 1875. Bd. 65. S. 64 .

${ }^{3}$ ) Berl. klin. Wochenschr. 1877. No. 34. 
wie die der Nierengeschwïlste. In dem Falle von Eberth fanden sich dagegen wirkliche Metastasenbildungen in Form von linsenbis bohnengrossen markigen Rrötchen, und diese enthielten ebenfalls Muskelfasern. - In dem meinigen waren, wie erwähnt, metastatische Knoten in der Leber von rein sarcomatöser Beschaffenheit vorhanden.

Was die Entstehung dieser Tumoren betriff, so bat bereits Eberth "die Annahme einer Aberration von Muskelelementen und eine spätere Wucherung derselben" nicht für allzu gezwungen gehalten, indem er auf den Reichthum des Wolff'schen Körpers an Keimzellen für Muskel- und Bindegewebe hinweist. Cobnheim nahm (unabhängig von $\mathbf{E} b$ erth) eine fehlerhafte Abschnúrung von Muskelkeimzellen, welche sich der ersten Urnierenanlage beimischten, an, und in seinem neuen Werke gelten ihm die in Rede stehenden Geschwülste als kräftige Stützen seiner Theorie der Geschwulstbildung überhaupt ${ }^{\mathbf{y}}$ ). - - In der That dürfte es vor der Hand schwer sein, eine andere, befriedigende Erklärung an die Stelle dieser Hypothese zu setzen.

Die bisher beobachteten Fälle von Geschwülsten aus quergestreiften Muskelfasern - von denen in der Literatur einige zwanzig Fälle aufgezeichnet sind - kann man in mehrere Abtheilungen ordnen. Die eine Reihe umfasst solche, welche durch Wucherung der normalen quergestreiften Musculatur entstanden sind. Seitdem die streitige Frage von der Regeneration des Muskelgewebes dahin entschieden ist, dass eine solche statt hat, und zwar sehr wahrscheinlich durch Proliferation der Muskelelemente selbst, seitdem liegt die Möglichkeit nahe, dass diese Proliferation das gewöhnliche Maass überschreiten und selbständige Wucherung; also geschwulstartige Neubildung veranlassen kann. Dahin gehören die Fälle vom Herzen $^{2}$ ) und die beiden von $\mathrm{Buhl}^{3}$ ) beschriebenen, wahrscheinlich - wenigstens nach der. Ansicht von klebs - auch der von Fr. Kaschewarowa-Rudnewa beschriebene Fall aus der Vagina ), sowie die Weber'sche Makroglossie ${ }^{5}$ ).

1) Vorlesungen über allgem. Pathologie. S. 643.

2) v. Recklinghausen, Monatsschr. f. Geburtskunde Bd. XX. 1862. S. 1. Virchow, Dieses Archiv Bd. 30. 1864. S. 468 und Bd.35. 1866. S. 211.

3) Zeitschrift f. Blologie Bd. I. 1865. S. 263.

4) Dieses Archiv 1872. Bd. 54. S. 63. - Klebs, Handbuch I. S. 961.

) Dieses Archiv Bd. 7. S. 115. 
Eine zweite Reihe wird gebildet durch die Fälle, in welchen quergestreifte Muskelfasern auftreten in Organen, welche normaler Weise dieselben ganz entbehren. Das vollkommen Fremdartige dieser Art Neubildungen gab schon in den ersten Fällen Anlass zu der Annabme, dass es sich hier um teratoide Bildungen handelte, eine Annahme, welche durch die sehr gemischte Natur einiger dieser Geschwülste unterstützt wurde ${ }^{1}$ ). Ueberdies kamen mehrere dieser Fälle vor in den Geschlechtsdrüsen, welche auch von anderweitigen offenbar teratoiden Neubildungen besonders heimgesucht sind [Rokitansky ${ }^{2}$ ), Virchow ${ }^{3}$ ), Billroth ${ }^{4}$, Senftleben ${ }^{5}$ vielleicht auch der Fall von Billroth aus der Mamma $\left.\left.{ }^{6}\right)\right]$.

Es giebt jedoch noch eine kleine Anzahl von Beobachtungen, in welchen es kaum anders möglich ist, als dass eine. Heteroplasie von quergestreiften Muskeln aus glatten Muskelfasern stattgefunden hat. 0 . Weber, welcher einen dieser Fälle beschreibt, ging sogar noch weiter, indem er die heterologe Entstehung von Muskelfasern sogar von den Bindegewebs- resp. Granulationszellen annahm [worin ihm Kolessnikow ${ }^{7}$ ) in seinem, wie mir scheint, nicht ganz klar gestellten Falle von melanotiscbem Rhabdomyom folgte]. Wenn der Fall von Anderson und Oedmannson nach Klebs ${ }^{8}$ ) eine andere Deutung zulässt, so spricht doch der von Bostroumoff und Eckert, welchen Kolessnikow anführt, unzweifelhaft für eine solehe Umbildung der glatten Muskelfasern des Uterus.

Eberth ${ }^{9}$ ) hat an der Oberfläche der menschlichen Niere ein Netzwerk von glatten Muskelfasern entdeckt und in der bezüglichen Mittheilung bemerkt er, dass sich aus diesem Vorkommen „für manche Myosarcome der Niere eine sehr einfache Erklärung ergebe“. An sich ist es jedoch sehr unwahrscheinlich, dass diese massen-

1) Virchow, Teratoma myomatodes mediastini. Dieses Archiv 1871. Bd. 53. S. 441.

$\left.{ }^{2}\right)$ Lehrbuch der path. Anat. I. 1855. S. 189.

3) Archiv f. path. Anat. Bd. VII. 1854. S. $13 \%$.

4) Archiv f. path. Anat. Bd. VIII. 1855. S. 433.

5) Archiv f. path. Anat. Bd. XV. 1858. S. 336.

$\left.{ }^{6}\right)$ Archiv f. path. Anàt. Bd. XVIII. 1860. S. 70.

7) Archiv f. path. Anat. Bd. LXVIII. 1876. S. 554.

8) Handbuch 1. S. 963.

${ }^{\natural)}$ Centralbl. f. d. med. Wissensch. 1872. No. 15. 
haften Neubildungen von Muskelsubstanz von den spärlichen normalen Elementen berstammen, auch spricht dagegen die Art des Wachsthums dieser Geschwülste, welche die Niere gewissermaassen von innen her auseinanderdrängten, ferner die gemischte Natur der Neubildung. Andererseits fehlt es für eine heteroplastische Entstehung von der normalen Anlage her, welche nach dem oben Gesagten nicht ohne Beispiel wäre, für die in Rede stehenden Fälle noch an hinreichendem Beweis, indess bleiben die oben (S. 293) erwähnten Uebergangsformen von glatten zu quergestreiften Fasern sehr bemerkenswerth ${ }^{1}$ ).

Iı Bezug auf die sarcomatösen Theile lässt Cohnheim unentschieden, ob die kleinen rundlichen Zellen als gewucherte Elemente des Bindegewebes oder ob sie als Vorbereitungsstadium der musculösen Gebilde zu betrachten seien, er hält jedoch das Letztere für wabrscheinlich. Es würde sich in diesem Falle also um indifferente Bildungszellen handeln.

Ein allmählicher Uebergang dor dicht gedrängten Rundzellen zu Bindegewebselementen lässt sich nun wobl nachweisen, indess gelang es mir nicht, unzweifelhafte Uebergangsformen zu jungen Muskeifasern zu finden; wie erwähnt, treten diese zerstreut in den zelligen Haufen auf, aber den jungen Spindelzellen ist ihre wahre Natur schwer anzusehen. - Das Fehlen der musculösen Elemente in den Leberknoten dient ebenfalls nicht zur Bestätigung jener Auffassung, indess ist es sehr wohl denkbar, dass bei längerem Bestehen sich auch hier Muskelfasern entwickelt haben wïrden wie in den Metastasen im Eberth'schen Falle und in dem kleineren Nierenknoten bei Cohnheim.

Primäre Geschwülste der Nieren kommen im kindlichen Alter im Vergleich mit den späteren Jahren verhältnissmässig häufig vor. Rohrer ${ }^{2}$ ) findet unter 96 aus der Literatur gesammelten Fällen

1) Von besonderem Interesse ist ein leider nicht zur Section gekommener Fall, welchen Ranke (Arch. f. klin. Chirurgie Bd. XXI. S. 692) erwähnt; es handelte sich um einen sehr umfangreichen Tumor der Niere bei einem 3jährigen Kinde, welcher sich auf Grund der Untersuchung eines zum Zweck der Diagoose exstirpirten Stückchens als Leiomyom erwies. Nach mündlicher Mittheilung des Herrn Dr. Ranke bestand dasselbe aus langen spindelförmigen Zellen mit stäbchenförmigen Kernen.

2) Das primäre Nierencarcinom. Monographie. Zürich 1874. 
von sogenanntem primären Nierenkrebs, bei welchen das Alter angegeben ist, 37 Fälle bei Kindern unter 10 Jahren und davon betrafen 16 Kinder unter zwei Jahren.' Dieses Verhältniss spricht augenscheinlich für die congenitale Natur dieser Geschwülste, wenn man die letztere auch nicht mit Gohnheim bereits für alle wabren Geschwülste anzunehmen geneigt ist. Wenn auch jene Tumoren wahrscheinlich grösstentheils wirkliche Carcinome resp. Adenome waren - die bezügliche Arbeit berücksichtigt histologische Verhältnisse leider so gut wie gar nicht, und bei den älteren Fällen fehlt es überhaupt an näheren Angaben - so ist ein Theil jener Fälle vermuthlich von der Natur des vorliegenden gewesen. Die äusserliche Uebereinstimmung erstreckt sich z. B. in Rohrer's Fall 21 bis auf das Vorhandensein der gefässreichen Excrescenzen im Nierenbecken, wie in den Fällen von Eberth und mir. Das Vorkommen epithelialer Gebilde in dem vorliegenden Falle deutet darauf hin, dass in etwaigen ähnlichen Beobachtungen auf Mischformen mit Carcinom resp. Adenom zu achten sein wird ${ }^{\mathfrak{1}}$ ).

In Bezug auf das mikroskopische Verhalten der musculösen Elemente stimmt die vorliegende Geschwulst ganz überein mit den von den früheren Beobachtern beschriebenen. Es handelte sich stets um Muskelfaseru, welche auf einer embryonalen Stufe der Entwickelung stehen bleiben; in allen Fällen waren die Fasern dünn und besassen kein Sarcolemm. Auch die jüngeren Entwickelungsstufen sind ziemlich übereinstimmend beschrieben worden. Nur in den drei am Herzen vorgekommenen Fällen verhielten sich die Elemente etwas abweichend. Anders verhielten sich ferner die quergestreiften Zellen in den von $B i l l \mathrm{rth}^{2}$ ) und von $\mathrm{Lambl}{ }^{5}$ ) beschriebenen Geschwülsten der Exlremitäten, welche deshalb schon

1) Nach Beendigung dieser Arbeit veröffentlichten Kocher und Langhans (Deutsche Zeitscbr. f. Chirurgie Bd. IX, Hft. 3 u. 4) einen Fall von Adenosarcom der Niere, in dessen Stroma quergestreifte Muskelfasern eingelagert waren. Es war also hier das umgekehrte Verhältniss der Geschwulstelemente vorhanden, wie in meinem Falle, ein Unstand, welcher für die Entstehungsgeschichte dieser Geschwülste in sofern von Wichtigkeit ist, als dieselben wenn überhaupt, so doch jedenfalls nicht allein auf die Abschnürung von Muskelelementen bezogen werden können, wie sich aus obigem bereits vermathen liess.

2) Dieses Archiv Bd. 1X. 1856. S, 172.

3) Aus dem Franz-Joseph Kinderspitale in Prag. 1860. S. 191. Taf. XII. 
früher von den in Rede stehenden mit Recht getrennt worden $\operatorname{sind}^{3}$ ).

Die Entwickelungsformen, welche man in der vorliegenden Geschwulst neben einander beobachten kann, gleichen ganz denen der normalen Muskeln. In dieser Beziehung möchte ich noch einmal den Umstand hervorheben, dass die Kerne stets seitlicb, resp. a uf den Fasern und niemals in denselben liegen. Niemals finden sich vollkommene Röbren mit einer quergestreiften Rinde und protoplasmatischer kernführender Mitte, wie dies Ranvier ${ }^{2}$ ) angiebt. Anch im normalen embryonalen Muskel habe ich nur jenes Verhalten beobachtet. Ich bin daher geneigt, dies Verhalten der Kerne als ein gutes Characteristicum der jungen quergestreiften Muskelfaser anzusehen.

Eine Fráge, deren Aufklärung noch immer nicht gesichert ist, ist die Entstehung des Sarcolemma, welches die einen bekanntlich für bindegewebiger Natur, die anderen für umgewandelte Zellenmembran erklären. Ein ausgebildetes Sarcolemma fand sich nun an unseren Fasern nicht, indess kann ich mich der Vermuthung nicht entziehen, dass die oben erwähnten zarten Häutchen, welche sich über die Kerne spannen und sich oft eine Strecke weit an der Faser verfolgen lassen, mit der Bildung des Sarcolemma in Beziehung zu setzen sind.

Klebs ${ }^{3}$ ) vermisst bei den früher beschriebenen quergestreiften Myomen den Nachweis der Doppelbrechung zur Sicherung der Diagnose; er selbst führte diesen Nachweis an der von Fr. Kaschewa a ow a beschriebenen Geschwulst.

Kolessnikow wies ebenfalls die Doppelbrechung der quergestreiften Spindelzellen in seinem Falle nach; in dem meinigen ergab die Untersuchung mit einem guten Schröder'schen Instrument ebenfalls die doppelbrechende Beschaffenheit der Querstreifen. Muskelfasern, welche einen quergestreiften Saum und glatte Mitte besassen, zeigten im dunklen Gesichtsfelde bei gekreuzten Nicols jederseits einen aus hellen und dunklen Abschnitten bestehenden Rand, während der mittlere Theil dunkel blieb. Es wird durch dies Verhalten wohl die Uebereinstimmung mit normalen quergestreiften Muskelfasern noch bestätigt, indess halte ich den dia-

1) Virch ow, Geschwülste. III. S. 106.

2) Traité technique d'histologie. p. 513 .

3) Handbuch S. 963 . 
gnostischen Werth desselben nicht für so bedeutend. Ausgebildete Muskelfasern sind stets als solche erkennbar; wenn es sich dagegen um die Entscheidung der Frage handelt, ob undeutlich quergestreifte spindelförmige Elemente musculöser Natur sind oder nicht, so dürften doch selbst gute Polarisationsapparate im Stiche lassen. Wenigstens gelingt der Nachweis der Doppelbrechung an einzelnen Fibrillen normaler Muskeln nur sehr unvollkommen. Ein negatives Ergebniss der Untersuchung ist daher nicht beweisend für die nicht musculöse Beschaffenheit.

\section{XVII. \\ Kleinere Mitheilungen.}

1.

Ueber die arzneiliche Anwendung des Jodbleis 1. s. w.

Von Dr. J. E. Schönfeldt in Dorpat.

Nachtrag zu diesem Archiv Bd. 65.

Die Veranlassung zu diesem Nacbtrage ist die vermuthlich nicht allgemein bekanot gewordene Dissertation von Dr. Fr. Berg, Beiträge zur Pharnakologie und Toxikologie der Jodpräparate, Dorpat 1875. Aus den Versuchen dieses Autors geht herror, dass die Krankbeitserscheinungen und Sectionsbefunde nach Vergiftungen mit verschiedenen Jodpräparaten wesentliche Unterschiede zeigen, wie das namentlich bei den Vergiftungen mit Jodnatrium und Jodtinctor, gegenüber denen mit Jodblei, in die Augen fällt.

Schon ältere Beobachtungen hatten gelehrt, dass in einem Falle, wo zur Heilung eines Cystovariums eine Lösung von einer Dracbme Jodkalium, in gleichen Theilen Jodtinctur und Wasser, zehn Unzen, eingespritzt und vier Minuten darauf wieder entleert worden war, unter Eiskälte und bläulicher Färbung des Gesichts und der Hände, kleinem kaum füblbarem Pulse, Erbrechen eingetreten war und der Puls sich erst am zweiten Tage darnaclı wieder gehoben hatte, indem er unter Schwinden der Cyanose, heisser hoher Röthung des Gesichts, weicher und voller wurde. Erbrechen aber und eine gleichzeitig eingetretene Anürie dauerten bis zum 7. Tage, an welchem der Tod erfolgte, fort, während welcher Zeit der Magen, unter angeblicher Exfoliation seiner Labdrüsen, einen grossen Theil des aufgenommenen Jods eliminirt batte. (Dr. E. Rose, das Jod in grossen Gaben, Studien an Eierstockeinspritzungen, Dieses Arch. Bd. 35.) 